Théologiques

Théologiques

\title{
Penser le discernement avec M.-D. Chenu, J.-M. R. Tillard et C. Theobald
}

\section{Notes sur une recherche en cours}

\section{Ignace Ndongala Maduku}

Volume 22, numéro 2, 2014

Le discernement spirituel

URI : https://id.erudit.org/iderudit/1035690ar

DOI : https://doi.org/10.7202/1035690ar

Aller au sommaire du numéro

\section{Éditeur(s)}

Faculté de théologie et de sciences des religions, Université de Montréal

\section{ISSN}

1188-7109 (imprimé)

1492-1413 (numérique)

Découvrir la revue

\section{Citer cette note}

Ndongala Maduku, I. (2014). Penser le discernement avec M.-D. Chenu, J.-M. R. Tillard et $\mathrm{C}$. Theobald : notes sur une recherche en cours. Théologiques, 22(2), 167-178. https://doi.org/10.7202/1035690ar

\section{Résumé de l'article}

La notion de discernement connaît aujourd'hui un intérêt particulier comme en fait foi son utilisation fréquente dans le langage courant et ses références dans bon nombre de publications du magistère et des théologiens. Partant des travaux de M.-D. Chenu, J.-M. R. Tillard et C. Theobald, cet article s'intéresse à ses significations et ses implications théologiques. 


\title{
Penser le discernement avec M.-D. Chenu, J.-M. R. Tillard et C. Theobald
}

\section{Notes sur une recherche en cours}

\author{
Ignace Ndongala Maduku* \\ Théologie et sciences des religions \\ Université de Montréal (Canada)
}

Depuis le dernier concile, la notion de discernement connaît un regain d'intérêt comme en fait foi son utilisation fréquente dans le langage courant et ses références dans bon nombre de publications du magistère et des théologiens. Il y est question du discernement spirituel, moral, éthique ou pastoral. La notion est aussi appliquée aux esprits, aux charismes, au sensus fidelium, au magistère, aux théologiens, aux communautés, etc. D'aucuns y recourent pour rendre le latin perscrutari (voir Gaudium et Spes (GS) 4a ${ }^{1}$. Le sens de la notion de discernement - qu'on retrouve aussi dans Presbyterorum Ordinis 17 et Gaudium et Spes 11, 44 et $52^{2}$ - varie selon les auteurs. Il convient dès lors d'en cerner les contours et d'en dégager les significations.

C'est à cette tâche que va s'atteler cette réflexion qui prend appui sur trois théologiens francophones retenus à cause de leurs travaux sur le

* Ignace Ndongala Maduku est professeur invité à l'Institut Lumen Vitae (Bruxelles) et chargé de cours et doctorant à la Faculté de théologie et de sciences des religions de l'Université de Montréal. Son projet de recherche doctorale porte sur les autoritarismes étatiques et ecclésiastiques en République démocratique du Congo. Ses recherches actuelles portent sur l'analyse transversale de la religion dans l'espace public à partir de la corrélation entre ecclésiologie, anthropologie, sociologie et politique. Il a récemment publié (2014) M. Moerschbacher et I. Ndongala Maduku, dir., Culture et foi dans la théologie africaine. Le dynamisme de l'Église catholique du Congo Kinshasa, Paris, Karthala.

1. Cette équivalence est aussi consacrée par la Commission théologique internationale (CTI) $\left(2012, \mathrm{n}^{\circ} 56\right)$.

2. Sur les occurrences conciliaires du terme de discernement, lire Ochoa $(1967,150)$ et Delhaye, et al. (1974, 194). 
concile Vatican $\mathrm{II}^{3}$. Les lignes qui suivent rendent compte de trois types d'approche qui éclairent avec des touches précises la notion du discernement ${ }^{4}$. Après avoir explicité la compréhension du discernement comme lecture des signes des temps chez M.-D. Chenu, nous développons le discernement du sens de la foi dans l'acception de J.-M. R. Tillard. Nous présentons enfin le discernement comme processus spirituel de formation de la vraie vie chez C. Theobald.

\section{M.-D. Chenu: le discernement comme auscultation des signes des temps en Église et par l'Église}

L'intelligence du discernement chez Chenu s'éclaire à partir de son approche des signes des temps. Cette dernière est relative au développement que le dernier concile consacre à cette expression. Bien qu'elle ne soit pas un obiter dictum, Chenu considère l'expression signum temporum comme l'une des formules les plus significatives du dernier concile. En effet, elle est à ses yeux "une catégorie constitutionnelle». Aussi, dans la Constitution Gaudium et spes, elle décide des lois et des conditions de l'évangélisation (Chenu 1967a, 225). Au dernier concile, l'expression «signes des temps» est adossée à la théologie des valeurs humaines. Elle donne lieu à des débats intenses qui aboutissent dès 1963 à son adoption définitive. On trouve dans le texte de la Constitution Gaudium et spes une liste non exhaustive des signes des temps pour le monde de ce temps.

L'expression "signes des temps » assigne aux événements humains une valence historique dont l'intelligence nécessite l'apport des sciences humaines. Elle module le style de la Constitution Gaudium et spes. Moins doctrinale et légale, cette dernière procède de manière inductive et part du

3. Rappelons pour mémoire que Chenu et Tillard ont participé au dernier concile respectivement comme consultant et expert. Quant à Theobald, il est l'un des meilleurs historiens et commentateurs contemporains du concile Vatican II.

4. Les réflexions qui suivent participent du projet de recherche auquel nous a associé le professeur Fabrizio Vecoli. Les résultats de nos investigations comportaient deux volets. Le volet heuristique a consisté à repérer le terme du discernement dans les documents théologiques et canoniques de l'Église, dans les déclarations du magistère et dans la réflexion théologique. Le travail heuristique s'est fait en deux moments. D'abord, en consultant les tables et index des différentes éditions françaises du concile Vatican II et du Code de droit canonique. Ensuite, en analysant les index de langue latine consacrés aux 16 documents du concile Vatican II et au Code de droit canonique. Le volet épistémologique et herméneutique a dégagé le sens et la signification du discernement dans les travaux de trois théologiens francophones dont les écrits remontent à la période postconciliaire. 
constat que les événements humains sont des «signes» inscrits en quelque sorte dans le mouvement de l' «histoire». De là, les pères conciliaires insistent sur la nécessité de discerner leurs valeurs. Chenu aide à faire comprendre que l'expression "signes des temps" intègre les conjonctures temporelles qui concourent au développement des ressources et des facultés de la nature de l'homme (Chenu 1967b, 98s). C'est avec cette connotation que l'expression apparaît dans la bulle Humanae salutis (25 décembre 1961). Nous pouvons donc dire qu'avec cette expression, l'historicité de l'homme est convoquée, et amarrée à l'anthropologie.

Chenu distingue trois sortes de signes: naturels, conventionnels et historiques (Chenu 1966, 30-40). Selon le théologien dominicain, par "signes des temps" il faut entendre "des phénomènes généralisés, enveloppant toute une sphère d'activités, et exprimant des besoins et les aspirations de l'humanité présente" (Chenu 1967b, 108). Il s'agit "des matériaux dans lesquels le peuple de Dieu discerne les signes de la présence et de la conduite de Dieu, non pas par quelque interprétation miraculiste, mais par l'intelligence de leur densité humaine» (Chenu 1967b, 108). Ces matériaux déclenchent une prise de conscience du peuple de Dieu qui est constitutive du signe. Comme il le précise, c'est un "acte psychologique qui ne procède pas d'une déduction menée à partir d'une théorie, d'une doctrine préalable, mais qui émane d'une perception provoquée par un engagement, dans une praxis» (Chenu 1967a, 211). Une telle prise de conscience relève d'un discernement susceptible d'instaurer une rupture dans la continuité du temps humain. Il s'agit donc des "phénomènes qui, par leur généralisation et leur grande fréquence, caractérisent une époque, et par lesquels s'expriment les besoins et les aspirations de l'humanité présente» (Chenu 1967a, 208). De tels faits sont des événements qui embrassent tout un cycle de vie collective, saisissent une génération, un peuple ou une civilisation. Ils concourent à l'évolution de l'humanité, à la construction du Royaume de Dieu. Chenu accorde une acception positive à l'expression "signes des temps» et lui assigne un soubassement axiologique, ce qui exclut des événements comme l'athéisme, le racisme, etc.

Il s'ensuit que les faits humains et événements renvoient à une réalité tout autre. En dépit de leur banalité, ils portent en eux les appels et les sollicitations de l'Esprit. Ainsi compris, ils constituent un lieu théologique ${ }^{5}$.

5. Pour une critique de l'idéologisation des signes des temps, lire Valadier (1971). L'auteur ne reconnaît que le Christ comme seul et unique signe des temps. 
Tâche pour le croyant inspiré par l'Esprit de les scruter pour, d'une part, y lire les besoins et les aspirations de l'humanité et, d'autre part, en avoir l'intelligence. Ce qui entraîne pour l'Église l'exigence de tenir compte de l'historicité, d'habiter le temps et d'être "présente" aux temps (Chenu 1967b, 110). Au regard de ce qui précède, Chenu rejette toute position dualiste face aux événements du monde et prend en compte les valeurs profanes. Cela fonde la posture qui considère les événements comme des "pierres d'attente», des "puissances obédientielles ». C'est dire combien les événements sont porteurs en germe des virtualités de progrès technique, des innovations économiques, des conditionnements sociaux, des régimes politiques, des échanges culturels et des mentalités psychiques (Chenu 1967 b , 108). Les valeurs du monde sont dès lors comprises comme des virtualités porteuses des "semences" de vérité, des expressions de la volonté de Dieu, des ressources possibles en préparation de l'Évangile. L'insertion dans le tissu unique du Logos venu dans l'histoire donne aux événements une autre densité qui requiert le discernement. Chenu, dont les travaux sont une référence incontournable pour l'interprétation de l'expression "signes des temps", traduit perscrutari de GS 4 par discerner. Comment comprendre ce terme?

D'après l'approche scripturaire, discerner, c'est savoir "vérifier toute chose et retenir ce qui est bon» $(1 \mathrm{Tm} 5,21)$. Ce sens, qu'on retrouve chez Jean XXIII, renvoie à la compréhension d'un discours ou de tout propos et peut être assimilé à un déchiffrement, à une analyse des signes des temps. Une telle analyse requiert une estimation et un jugement de valeur émanant du génie de l'homme. Dans la ligne du numéro 11 de Gaudium et spes, elle ne peut être que l'œuvre de l'Esprit: elle est une lecture des événements par un pressentiment global des échéances successives qui en éclairent le destin (Chenu 1967b, 107). Il sied de préciser que selon Chenu, cette lecture est exempte de toute visée apologétique et de toute portée de surnaturalisation. Elle respecte les événements dans leur singularité et factualité, tout en les appréhendant comme porteurs des appels de l'Évangile et sujets de la grâce (Chenu 1967b, 109). En conséquence, le discernement est une lecture des desseins de Dieu, du Dieu créateur et du Dieu rédempteur, du Dieu meneur de l'histoire sainte par la foi en éveil (Chenu 1967b, 113). Chenu parle d' "ausculter» les événements selon leurs lois propres, avec intelligence et émotion. Telle est la tâche du prophète qui, à la différence du docteur, se révèle plus réaliste " parce qu'il lit dans l'histoire. Il perçoit les signes des temps, au-delà des énoncés des principes»(Chenu 1967b, 109). 
La mention des prophètes laisserait croire que le discernement est réservé à une caste. Rien de tel, car Chenu l'étend à toute l'Église, aux chrétiens en Église. C'est par ailleurs ce qu'enseignent les paragraphes 11 et 44 de Gaudium et spes. Le discernement requiert de l'Église entière qu'elle observe les signes dans leur actualité et qu'elle demeure présente à ces temps. Une manière de réaliser cette requête est pour l'Église de chercher en positif ou en creux, au cœur des événements, dans le devenir humain, les appels et sollicitations de l'Esprit. Puisque l'histoire est le théâtre des événements, elle devient un lieu théologique. Les fidèles qui vivent dans le monde (GS 44) sont dès lors professionnellement qualifiés pour exercer le discernement et retenir ce qui relève de Dieu et est conforme avec l'Évangile - ce qui touche aux critères du discernement. Pour Chenu, discerner, c'est s'inscrire, et inscrire son action dans la ligne du Royaume de Dieu à construire. En d'autres termes, c'est donner une dimension collective aux critères psychologiques de la théologie classique selon la mesure des événements à la taille de l'humanité nouvelle (Chenu 1967a, 217).

\section{J.-M. R. Tillard: le discernement comme instinctus de la communauté croyante}

Pas plus que ne le fait Chenu, Tillard n'aborde pas de front la question du discernement. Il la garde en filigrane de ses développements sur le «sensus fidelium» et sur la relation entre la fonction de "magistère» de la hiérarchie et les intuitions, suggestions et initiatives des fidèles. Il décèle une tension, d'une part, entre les prises de position officielles et la conscience spontanée des fidèles et, d'autre part, entre les exigences de la "foi savante» et les besoins de la «foi populaire». Leur discordance lui suggère une alternative qu'il formule sous forme d'une question: «Doit-on y voir un fait à déplorer, un manque grave d'obéissance mettant en cause la santé des églises [...], ou au contraire un agent de vitalité, préparant l'ouverture de l'Église aux exigences des temps à venir, et exprimant le vouloir mystérieux de l'Esprit?" (Tillard 1976, 10). D'aucuns, optant pour la deuxième partie de l'alternative, considèrent l'initiative des fidèles comme "la volonté du Seigneur» (Tillard 1976, 10). Faut-il cependant en dire autant de certaines manifestations de la foi populaire qui rebutent «le théologien spécialiste de la foi savante " ?, ainsi qu'il s'interroge (Tillard 1976, 11). La réponse à son interrogation le conduit à des élaborations sur le sensus fidelium. 
Il entend par cette expression l'attitude spontanée et la perception instinctive des fidèles qui n'appartiennent pas à la hiérarchie (Tillard 1976, 16). L'évocation des bulles Ineffabilis ( 8 décembre 1854) de Pie IX et Munificentissimus Deus (1 ${ }^{\text {er }}$ novembre 1950) de Pie XII, ainsi que de la lettre Deiparae Virginis (1 ${ }^{\mathrm{er}}$ mai 1946), lui permet d'établir que les interventions des papes prennent leur point de départ dans une tradition pratique qui rend compte d'une "singularis catholicorum antistitum et fidelium conspiratio".

On retrouve aussi l'acception du sensum fidelium comme accord remarquable des évêques et des fidèles catholiques chez le père Perrone et le cardinal Newman. Elle ne s'écarte pas de l'approche de Melchior Cano, selon qui «le sensus fidelium est un des éléments essentiels du sensus ecclesiae, une des fibres principales qui tissent la vie de foi du peuple de Dieu comme tel» (Tillard 1976, 16). Le sensus fidelium requiert donc l'authentification et la garantie du magistère. Tillard le souligne: bien que son autorité ne vienne pas des fidèles, le magistère tire du sensus fidei sa crédibilité. Il y a dès lors complémentarité, une adéquation plus ou moins grande entre ce qui se vit et s'exprime, dans le peuple de Dieu en son ensemble, et l'action spécifique du magistère, dont l'acte de définition dogmatique s'articule à une vérité vécue (Tillard 1976, 20). Tillard en conclut que le magistère ne constitue pas une autorité surajoutée à celle de la parole de Dieu, mais demeure un service de discernement de ce qui se vit dans le peuple de Dieu sur la base de l'accueil de la parole de Dieu et de la puissance de l'Esprit (Tillard 1976, 39).

En définitive, la nature et la fonction du sensus fidelium se comprennent à l'aune de l'action de l'Esprit-Saint dans le peuple de Dieu. Tillard reconnaît la présence des charismes et des fonctions au corps ecclésial tout entier. Il s'écarte de la vision descendante qui autonomise la hiérarchie et lui accorde le monopole de la connaissance du mystère chrétien qu'elle posséderait en plénitude. En insistant sur la diversité et la complémentarité des rôles, Tillard n'est pas résolument infondé à requalifier religieusement les fidèles laïcs (Tillard 1976, 21-25). On peut donc dire qu'en vertu de l'action de l'Esprit-Saint, les fidèles portent en eux le sens de la vérité (Tillard 1976, 23). Ainsi compris, le sensus fidelium gît au palier de la vérité qui jaillit entre la Parole reçue et ce qu'elle devient par la puissance de l'Esprit pour le croyant qui essaie de s'y conformer (Tillard 1976, 24). Dès lors, il y a place aussi bien pour le magistère qui annonce, explicite la parole en vue de la perception globale du sens de la parole, que pour les fidèles qui en vivent. Comme il le dit: "au contenu des mots, 
l'expérience du Christ apporte des harmoniques qui en ouvrent la signification» (Tillard 1976, 25). À ce sujet, une précision importante est à noter: dans la perspective de Tillard, le sensus fidelium ne se confond pas avec une libre interprétation personnelle de l'Écriture. Il naît au contraire de la parole annoncée par l'Église, reçue dans l'Église et comprise par l'Église sur la base objective (Tillard 1976, 29). Il n'ouvre pas à un fondamentalisme stérile, mais suppose plutôt la consonance avec le magistère hiérarchique, car, comme il l'explicite, "s'il y a ministères de vérité (ou magistère), ce n'est qu'à l'intérieur de la communauté, tout entière dotée de l'Esprit de vérité» (Tillard 1987, 145).

Reste à qualifier cette perception de la foi qui, dans sa perspective, est l'œuvre de l'Esprit-Saint. S'agit-il d'une simple intuition ou suggestion des fidèles? Tillard ne le pense pas. Il évoque un flair spirituel qui habite les fidèles (Tillard, 1976, 25). Ce phronèma des fidèles dont parle Newman a déjà été évoqué par Thomas d'Aquin qui "comptait parmi les effets du don de foi le pouvoir de discerner, même sans instruction, l'enseignement authentique du frelaté» (Tillard 1976, 25). Ce discernement est aussi le fait de ceux qui scrutent profondément le donné révélé, le magistère grâce au charisme qui leur est propre (Tillard 1976, 39s). C'est ce charisme qui dispose le magistère à puiser dans la vie même du peuple de Dieu la réalité à discerner, à juger, à promulguer ou à «définir». On peut dès lors dire que, considéré du point de vue de la hiérarchie, le discernement consiste à préciser, expliciter, défendre, l'objet du flair du peuple de Dieu. Il requiert une symbiose entre l'instinctus de la communauté croyante et ceux qui y exercent un magistère (Tillard 1987, 145). Le discernement est enserré dans l'instinct du sensus fidelium. Ainsi naît une écoute mutuelle, un dialogue, une conspiratio des fidèles et des pasteurs (Tillard 1987, 145) qui correspond au sensus ecclesiae. L'approche de Tillard tient ensemble le sensus ecclesiae et le sensus fidelium tout en gardant une réciprocité, une dépendance mutuelle, une synergie entre la foi des fidèles et sa formulation par les théologiens et le magistère.

\section{C. Theobald: le discernement comme processus d'interprétation}

Dans un ouvrage remarqué publié par les Éditions du Cerf en 2007, Theobald se lance dans la compréhension du christianisme comme style. Il y consacre un chapitre à la théologie comme discernement de la vie authentique (Theobald 2007, 413-437). En rendant compte de la manière ignatienne de faire la théologie, Theobald présente la tradition de la 
communauté qui a pétri son expérience humaine et spirituelle. Le propos sur son appartenance à une tradition tient ensemble la théologie dite "scientifique ", l'expérience spirituelle et sa manifestation dans la littérature spirituelle (Theobald 2007, 414). Ancré dans les Exercices spirituels, le modus procendi in theologie propre à la Compagnie de Jésus ne s'accommode pas d'un corps doctrinal ni d'une structure intellectuelle bien déterminée.

Les exercices spirituels évoquent la théologie à travers la notion traditionnelle de "doctrine». Le contenu est corrélé à un processus de transformation ou de conversion qui constitue en soi un "style ", une "forme " (Theobald 2007, 418) dont les traces sont perceptibles dans l'Écriture du corpus textuel tout entier et la construction du corps apostolique, ainsi que les institutions spécifiques, notamment les institutions d'enseignement (Theobald 2007, 419). La doctrine vise donc le «bien des âmes» et ne peut être séparée de la "vie» (Theobald 2007, 418). Elle est inscrite dans le processus global de "formation", d'incorporation du jésuite. Mieux, elle est un "processus complexe de formation d'une vraie vie, mis en mouvement et inspiré par une lecture des Écritures et aboutissant à une véritable (re-) création de ses lecteurs» (Theobald 2007, 423). On le voit, le modus procendi jésuite est "une manière de procéder ", un "tour de main ", un «style », porteur d'une expérience humaine et spirituelle qui vise une «formation de la vraie vie» que Theobald rend par le concept de «vie authentique» (Theobald 1997, 388). C'est précisément, poursuit-il, ce style que nous visons ici en parlant de «la théologie comme discernement de la vie authentique». Dans la perspective de Theobald, la vie authentique nécessite la lecture des Écritures et une mise en rapport entre l'espace des Écritures et l'espace culturel et politique actuel (Theobald 2007a, 416; 2007b, 167-187). Il s'ensuit que la vraie vie exige inventivité et discernement. L'inventivité est perçue ici comme l'entrée dans l'expérience créatrice des fondateurs avec comme critère décisif l'authenticité unique de la figure de Jésus. De cette manière, elle ouvre à la diversité des itinéraires individuels et à une variété de styles communautaires.

L'approche de Theobald s'articule autour des textes fondateurs de la Compagnie de Jésus. Ces textes considèrent le discernement comme un processus spirituel dont la visée est «la formation d'une vraie vie». La lecture actuelle de l'Écriture sous la mouvance de l'Esprit est l'acte effectif de la formation d'une vraie vie. Le discernement pratique accorde une place importante à la fonction structurante de l'Écriture et à l'itinéraire de Jésus et de ses apôtres croisant les routes des hommes (Theobald 2007, 
436). Appliqué à l'individu, le discernement requiert de «s'orienter [...] en fonction d'une expérience de conformité ou de concordance entre l'enracinement de sa liberté en Dieu et l'organisation toujours unique de sa vie» (Theobald 1997, 386). Theobald conçoit le discernement tant pour l'individu que pour une collectivité. À juste titre, il l'applique aux évêques réunis au concile. Il parle du discernement collégial des signes des temps. Comme pour l'individu, ici aussi l'Écriture joue une fonction structurante. C'est sous son autorité unique que s'opère le discernement. Cette soumission n'aliène cependant pas les motions de l'Esprit ni ne nivelle les différences des points de vue, ce qui permet au principe synodal et à la collégialité épiscopale d'être effectifs.

D'après son acceptation, le discernement est un processus d'interprétation balisé par les trois mots clés de Gaudium et spes 11 : événements, exigences et requêtes. Ce processus d'interprétation s'est déployé au concile Vatican II sous forme d'un long apprentissage de l'écoute de trois voix: d'abord la Parole de Dieu, ensuite ce qui est vraiment humain dans la société (GS 1) et enfin les voix complexes et discordantes de la «tradition ». Celle-ci est codifiée dans Unitatis redintegratio 11 qui prône la manière de chercher la vérité ensemble (Theobald 2007, 361). On comprend sans peine que cette manière collégiale de procéder est aussi une manière collégiale ou synodale de discerner les signes des temps sous l'autorité unique de la parole de Dieu (Theobald 2007, 362; 2012, 353-363) .

\section{Conclusion}

En conclusion à cette brève réflexion, on peut soutenir que les approches de Chenu, Tillard et Theobald ont résolument de spécifique de présenter le discernement respectivement comme un processus d'interprétation des signes des temps (Chenu), une perception du sens de la foi par les fidèles (Tillard) et un processus spirituel de formation de la vie authentique (Theobald). Les trois approches postulent une herméneutique des situations qui enrichit l'intelligence du discernement de l'apport de l'histoire considérée comme un «lieu théologique». Elles suggèrent l'ouverture au

6. Theobald approche la théologie des signes des temps comme un modus procedendi qui permet d'aborder la question de l'articulation de la particularité avec l'universalité. Cette articulation présuppose que l'on tienne compte de la pluralité des aires socioculturelles et que l'on situe l'accès à la vérité au terme d'un processus historicoculturel à la fois d'interprétation et de réception de la Révélation. Lire notamment Theobald (2006, 71-84, surtout les pages 72-75). 
pluralisme et à la particularité. L'attention aux contextes historiques et culturels éclaire la réalité sociale, politique et culturelle spécifique à chaque peuple, réalité qui entre en dialogue avec la tradition de foi. À tout bien considérer, ce dialogue donne à l'offre de la révélation des harmoniques neuves nées d'une actualisation vivante et intelligible de la Tradition.

L'approche du discernement par nos trois auteurs pose les jalons d'une réflexion sur le rapport entre le magistère et le sensus fidelium. Dans cette perspective, leurs analyses s'avèrent suggestives et font du discernement un outil herméneutique indispensable pour l'inculturation et la mission de l'Église dans le contexte actuel. Ayant pour horizon et autorité unique les Écritures, cette herméneutique est l'œuvre d'individus habités par l'EspritSaint ou du peuple de Dieu pris dans son ensemble (GS 44). Elle impose des critères que la Commission théologique internationale systématise: Jésus-Christ, l'apostolicité et la catholicité de la foi, la communion ecclésiale et l'édification de l'unité de l'Église (Documentation catholique 1990, 489-502). Il est certain, en tout cas que, dans cette tâche de discernement, une place remarquable est à assigner aux pasteurs et aux théologiens qui jugent, interprètent et discernent en conformité avec le magistère. Cela ne signifie pas, on le sait, qu'ils ont le monopole d'autorité. Au service du consensus fidelium, ils assurent la régulation institutionnelle du discernement dans l'Église catholique en demeurant à l'écoute du sensum fidelium. Ce n'est donc pas forcer la pensée de nos auteurs que de soutenir à la suite de Claude Geffré que cette régulation est "une autorégulation de toute l'Église comme corps vivant où chacun est appelé à jouer sa partition » (Geffré 1983, 103). On pourrait bien s'interroger, après beaucoup d'autres, sur l'application empirique de cette autorégulation dans l'Église catholique. Sous réserve d'en explorer de façon systématique les implications théologiques, ecclésiologiques, canoniques et pastorales, on peut présumer qu'elle entraîne une approche renouvelée de l'autorité et du statut de la vérité. Dans cette perspective, à la suite de nos auteurs, nous retenons que le discernement comporte une dimension herméneutique assortie aux harmoniques de la foi, certes, mais relative à la diversité des cultures et à la variété des contextes historiques du peuple de Dieu. C'est dire combien il requiert des apprentissages tant pour le peuple de Dieu et les théologiens que le magistère.

\section{Références}

Chenu, M.-D. (1966), Peuple de Dieu dans le monde, Paris, Cerf. 
(1967a), "Les signes des temps. Réflexion théologique », dans Y. Congar et M. Peuchmaurd, dir., L'Église dans le monde de ce temps. Constitution pastorale "Gaudium et spes", Paris, Cerf, p. 205225.

"Les signes des temps"(1967b), dans K. RAHner et al., L'Église dans le monde de ce temps. Constitution "Gaudium et spes". Commentaires du schéma XIII, Paris, Mame, p. 96-116.

Commission Théologique Internationale (1990), "L'interprétation des dogmes ", Documentation catholique, 2006, p. 489-502.

(2012), La théologie aujourd'hui: Perspectives, principes et critères, Paris, Cerf.

Delhaye, P., Gueret, M. et Tombeur, P. (1974), Concordance, Index, Listes de fréquence, Tables comparatives, Louvain, Cetedoc.

GefFré, C. (1983), "Le christianisme au risque de l'interprétation, Paris, Cerf.

OchоA, X. (1967), Index verborum cum documentis concilii Vaticani secundi, Roma, Commentarium pro religiosis.

Theobald, C. (2012), «Le concile Vatican II face à l'inconnu. L'aventure d'un discernement collégial des "signes des temps", Études, p. 353-363. (2007a), Le christianisme comme style. Une manière de faire la théologie en postmodernité, Paris, Cerf.

(2007b), «Lire les "signes des temps". À propos de la dimension sociale et politique de la foi ", Transmettre un Évangile de liberté, Paris, Bayard, p. 167-187.

(2006), «Zur Theologie der Zeichen der Zeit. Bedeutung und Kriterien heute", dans P. Hünermann, dir., Das Zweite Vatikanische Konzil und die Zeichen der Zeit heute, Freiburg, Herder, p. 71-84.

(1997), «Une manière ignatienne de faire la théologie. La théologie comme discernement de vie authentique ", Nouvelle revue théologique, 119/3, p. 375-396.

Tillard, J.-M. R. (1976), «Le sensus fidelium, réflexion théologique », Foi populaire, foi savante, Paris, Cerf (Cogitatio Fidei 87), p. 9-40. Cerf.

(1987), Église d’Églises. L’ecclésiologie de communion, Paris,

Valadier, P. (1971), "Signes des temps, signes de Dieu?", dans Études, 334, p. 261-279. 


\section{Résumé}

La notion de discernement connait aujourd'hui un intérêt particulier comme en fait foi son utilisation fréquente dans le langage courant et ses références dans bon nombre de publications du magistère et des théologiens. Partant des travaux de M.-D. Chenu, J.-M. R. Tillard et C. Theobald, cet article s'intéresse à ses significations et ses implications théologiques.

\section{Abstract}

The concept of discernment is experiencing particular interest as evidenced by its frequent use in everyday language and references in many publications of the magisterium and theologians. Starting from the work of M.-D. Chenu, J.-M. R. Tillard and C. Theobald, this article focuses on the meanings and theological implications of discernment. 